\title{
Resting Microglia Directly Monitor the Functional State of Synapses In Vivo and Determine the Fate of Ischemic Terminals
}

\author{
Hiroaki Wake, ${ }^{1,2}$ Andrew J. Moorhouse, ${ }^{1,3}$ Shozo Jinno, ${ }^{4}$ Shinichi Kohsaka, ${ }^{5}$ and Junichi Nabekura ${ }^{1,2,6}$ \\ ${ }^{1}$ Division of Homeostatic Development, National Institute of Physiological Sciences, Okazaki 444-8585, Japan, ${ }^{2}$ Core Research for Evolutional Science and \\ Technology, Japan Science and Technology Agency, Saitama 332-0012, Japan, ${ }^{3}$ Membrane Biophysics Laboratory, School of Medical Sciences, The \\ University of New South Wales, Sydney 2052, Australia, ${ }^{4}$ Department of Anatomy and Neurobiology, Graduate School of Medical Sciences, Kyushu \\ University, Fukuoka 812-8582, Japan, ${ }^{5}$ Department of Neurochemistry, National Institute of Neuroscience, Kodaira, Tokyo 187-8502, Japan, and \\ ${ }^{6}$ Department of Physiological Sciences, The Graduate School for Advanced Study (Sokendai), Hayama 240-0198, Japan
}

Recent studies have identified the important contribution of glial cells to the plasticity of neuronal circuits. Resting microglia, the primary immune effector cells in the brain, dynamically extend and retract their processes as if actively surveying the microenvironment. However, just what is being sampled by these resting microglial processes has not been demonstrated in vivo, and the nature and function of any interactions between microglia and neuronal circuits is incompletely understood. Using in vivo two-photon imaging of fluorescent-labeled neurons and microglia, we demonstrate that the resting microglial processes make brief $(\sim 5 \mathrm{~min})$ and direct contacts with neuronal synapses at a frequency of about once per hour. These contacts are activity-dependent, being reduced in frequency by reductions in neuronal activity. After transient cerebral ischemia, the duration of these microglia-synapse contacts are markedly prolonged $(\sim 1 \mathrm{~h})$ and are frequently followed by the disappearance of the presynaptic bouton. Our results demonstrate that at least part of the dynamic motility of resting microglial processes in vivo is directed toward synapses and propose that microglia vigilantly monitor and respond to the functional status of synapses. Furthermore, the striking finding that some synapses in the ischemic areas disappear after prolonged microglial contact suggests microglia contribute to the subsequent increased turnover of synaptic connections. Further understanding of the mechanisms involved in the microglial detection of the functional state of synapses, and of their role in remodeling neuronal circuits disrupted by ischemia, may lead to novel therapies for treating brain injury that target microglia.

\section{Introduction}

Microglial cells play an important role in various types of brain injury and disease, including ischemia, trauma and, Alzheimer's disease. Their substantial activation in response to these pathological states results in a transformation into an "amoeboid" morphology, migration toward the site of injury and release of various neuroactive compounds to ultimately result in neurotoxicity or neuroprotection (Vilhardt, 2005; Biber et al., 2007). In the absence of pathological insults, however, nonactivated "resting" microglia are far from dormant. In vivo two-photon imaging revealed that resting microglia are highly dynamic, extending and retracting their processes in a random manner and interspersed

\footnotetext{
Received Sept. 12, 2008; revised 0ct. 29, 2008; accepted Jan. 26, 2009.

This work was supported by the Core Research for Evolutional Science and Technology, Japan Science and Technology Agency, and Grants-in-Aid from Ministry of Education, Culture, Sports, Science, and Technology, Japan (J.N.). We gratefully acknowledge the support of Prof. H. Kasai at Tokyo University, Dr. T. Nemoto at National Institute for Physiological Sciences (NIPS) for the establishment of the in vivo two-photon imaging system, and the expert technical assistance of M. Yoshitomo, T. Ohba (NIPS), and Y. Hirakawa (Research Support Center, Graduate School of Medical Sciences, Kyushu University).

Correspondence should be addressed to Prof. Junichi Nabekura, Division of Homeostatic Development, Department of Developmental Physiology, National Institute for Physiological Sciences, 0kazaki 444-8585, Japan. E-mail: nabekura@nips.ac.jp.

D0I:10.1523/JNEUROSCI.4363-08.2009

Copyright $\odot 2009$ Society for Neuroscience $\quad$ 0270-6474/09/293974-07\$15.00/0
}

with brief static periods (Nimmerjahn et al., 2005). An injury stimulus resulted in a rapid and directed expansion/growth of these processes toward the site of injury and in response to a signaling cascade involving endogenous ATP release (Davalos et al., 2005) and $\mathrm{P}_{2 y 12}$ receptors (Haynes et al., 2005). The dynamic surveillance of the brain environment is so extensive. However, just what exactly is being sampled, or why the microglial processes show brief static periods, is not exactly clear. Such questions should be addressed in an intact brain because direct access, even opening of the skull, readily activates microglia (Xu et al., 2007).

Previous immunohistochemical and electron microscopic studies have revealed that activated microglia closely appose neurons, where they might have some role in apoptosis and remodeling of the nervous system. For example, the role of microglia has been investigated in the removal of synapses that occurs after nerve axotomy and during development (Blinzinger and Kreutzberg, 1968; Bruce-Keller, 1999; Bessis et al., 2007; Trapp et al., 2007). However, the dynamics of such interactions between intact/injured synapses and microglia has not been characterized. We hypothesized a close structural and functional association between microglial processes and synapses that can respond to the physiological and pathophysiological state of the local brain microenvironment. 


\section{Materials and Methods}

Description and preparation of transgenic mice used for imaging. All relevant experimental protocols were approved by the Ethics Review Committee for Animal Experimentation of the National Institutes for Natural Sciences. We used two different strains of transgenic mice derived from the C57BL/6 strain: a transgenic mouse in which enhanced green fluorescent protein (EGFP) was expressed under control of the microglia and macrophage-specific promoter (ionized $\mathrm{Ca}^{2+}$-binding adapter molecule 1; Ibal-EGFP mouse) (Hirasawa et al., 2005) and a mouse with sparse expression of cytoplasmic GFP in thalamic and cortical pyramidal neurons (Thy1 promoter GFP mouse, $\mathrm{M}$ line) (Feng et al., 2000). Transgenic mice, at 6-10 weeks after birth, were anesthetized with an intraperitoneal injection of ketamine $(0.13 \mathrm{mg} / \mathrm{g})$ and xylazine $(0.01 \mathrm{mg} / \mathrm{g})$. Body temperature was monitored with a rectal probe and maintained at $36-37^{\circ} \mathrm{C}$ using a heating blanket, except when investigating altered neuronal activity in which case it was lowered to $32^{\circ} \mathrm{C}$. The depth of anesthesia was assessed by monitoring the withdrawal reflex to pinching the footpad and also by monitoring respiration rate and vibrissae movements. For the ischemia model, only male mice were used. For transcranial (thin-skull) imaging, the scalp was gently separated, and the skull was first thinned with a high-speed drill under a dissecting microscope, before being gently scraped with a microsurgical blade to a final thickness of $\sim 30 \mu \mathrm{m}$. The thickness of the skull was estimated with measurements of the distance at which auto immunofluorescence of the skull was observed using two-photon imaging. A metal ring surrounding this region was attached to the skull with dental acrylic cement, and artificial mouse CSF was used to keep the thinned region moist during experiments.

In vivo two-photon microscopy. GFP-labeled constructs were imaged by a two-photon microscopy using a water-immersion objective lens $(60 \times$, NA1.1) at zoom of 1-2.5 (Olympus). A Ti-sapphire laser (MaiTai $\mathrm{Hp}$, Spectral Physics) was tuned to the excitation wavelength for GFP $(950 \mathrm{~nm})$. Time-lapse imaging of small cortical regions was performed by repeated acquisition of small fluorescence image stacks comprising $40-50$ focal planes, each with $0.5 \mu \mathrm{m}$ axial spacing. The photomultiplier tubes setting and excitation power $(<10 \mathrm{~mW})$ were kept constant during time-lapse imaging. The interval of free running scanning was $0.3-1.0 \mathrm{~s}$. Other images were obtained every $10 \mathrm{~min}$. The typical imaging depth was from 100 to $250 \mu \mathrm{m}$ from the pial surface.

Although both neurons and microglia had the same fluorophore (GFP), we were able to distinguish the fluorescence from microglia and neurons, attributable to their distinct morphologies and because of the motility of microglia compared with the static neurons, as now described in Materials and Methods. We cannot exclude the possibility that other nonlabeled neuronal elements were interspersed between the microglia and synapses; we confirmed direct contacts using electron microscopy.

Images were obtained in mice anesthetized with an intraperitoneal injection of ketamine $(0.13 \mathrm{mg} / \mathrm{g})$ and xylazine $(0.01 \mathrm{mg} / \mathrm{g})$. Such anesthesia is unavoidable in such in vivo experiments but may of course effect the cellular responses observed, such as the kinetics of microglial motility or frequency and/or duration of the microglia-synapse connections. Lendvai et al. (2000) reported no difference in dendritic spine motility in vivo in mice under ketamine or urethane anesthesia.

Pre-embedding immunoelectron microscopy. Five mice were used for immunoelectron microscopy, three controls and two that underwent middle cerebral artery (MCA) occlusion-induced ischemia (see below). They were deeply anesthetized with sodium pentobarbital $(100 \mathrm{mg} / \mathrm{kg}$ body weight) and perfused transcardially with PBS (pH 7.4) followed by a fixative containing $4 \%$ paraformaldehyde and $0.5 \%$ glutaraldehyde in $0.1 \mathrm{~m}$ phosphate buffer ( $\mathrm{pH} 7.4$ ). The brains were left in situ for $1-2 \mathrm{~h}$ at room temperature and were then cut coronally into $50-\mu \mathrm{m}$-thick serial sections using a vibrating microtome (VT1000S; Leica Microsystems). Slices were immediately frozen in liquid nitrogen (cryoprotected and freeze-thaw procedure), thawed and incubated overnight at $4^{\circ} \mathrm{C}$ with $1.0 \%$ BSA in PBS containing $0.05 \%$ sodium azide, then incubated with a rabbit polyclonal anti-Ibal antibody (1:5000; Wako Pure Chemical Industries) for $2 \mathrm{~d}$ at $4^{\circ} \mathrm{C}$. The sections were subsequently processed according to the avidine-biotinylated enzyme complex (ABC) method (Hsu et al., 1981), being fixed with $1.0 \% \mathrm{OsO}_{4}$ in $0.1 \mathrm{M} \mathrm{PBS}$ for $1 \mathrm{~h}$ at $4^{\circ} \mathrm{C}$, rinsed briefly in distilled water, and then stained with $1.0 \%$ aqueous uranyl acetate for $1 \mathrm{~h}$. They were then dehydrated in a graded series of ethanol, infiltrated in propylene oxide, and flat embedded in EponAraldite. Sections were examined and recorded photographically with a light microscope (Axioskop 2; Zeiss). Small areas containing the somatosensory cortex were identified and excised, remounted on a blank cylinder, and were cut into ultrathin sections $(70-80 \mathrm{~nm})$ using an ultramicrotome (Ultracut S; Leica Microsystems). The ultrathin sections were collected on 200 mesh grids and examined with a JEOL 2000EX electron microscope.

Basal $\mathrm{Ca}^{2+}$ fluorescent imaging. The loading of dye and the $\mathrm{Ca}^{2+} \mathrm{im}-$ aging were performed through a small open cranial window (open skull preparation). We used a modified version of the protocol of Ohki et al. (2005) to load cortical neurons with a calcium-sensitive dye under continuous visual guidance through the two-photon microscope. A total of $0.8 \mathrm{~mm}$ Oregon Green 488 BAPTA-1 AM (OGB-1 AM) was dissolved in DMSO with $20 \%$ pluronic acid (all from Invitrogen) and mixed in artificial CSF containing sulforhodamine 101 (SR101; Sigma). A standard patch-clamp pipette was filled with this solution and inserted into the cortex to a depth of 200-250 $\mu \mathrm{m}$ from the pial surface. OGB-1 AM and SR101 were pressure ejected (5-10 p.s.i, IM-300; Narishige) from the pipette for 40-80 s, giving an estimated ejection volume of $1 \mathrm{pl}$. After successful dye loading into the cell was confirmed by measuring fluorescent responses, the pipette was withdrawn and the craniotomy sealed with a glass coverslip. Calcium fluorescence in cortical cells was imaged with the two-photon Olympus microscope (Fluoview 1000) and fluorescence excited with the MaiTaiHP (Spectra Physics) mode-locked Ti:sapphire laser (power $15 \mathrm{~mW}$ ) at an excitation wavelength of $810 \mathrm{~nm}$. Excitation light was focused using $\times 20$ water-immersion objective $(0.9$ numerical aperture).

MCA occlusion. Focal cerebral infarction was produced by transient occlusion of the right MCA by a laser-induced photochemical reaction, using a modified procedure described previously (Yao et al., 2002). Mice were anesthetized by an intraperitoneal injection of ketamine $(0.13$ $\mathrm{mg} / \mathrm{g})$ and xylazine $(0.01 \mathrm{mg} / \mathrm{g})$. A midline neck incision exposed the right common carotid artery, which was loosely sutured. The thyroid gland was pulled aside, and the right jugular vein was exposed, carefully dissected from surrounding tissue and catheterized. The catheter was advanced into the superior vena cava, for intravenous administration of solution, and secured. The skin was cut between the right eye and ear, exposing the skull and right temporal muscle. The temporal muscle was retracted until the distal part of the right MCA was observed. The exposed tissues were kept moist with normal saline. A krypton laser (643Y-A01; Melles Griot), operating at a wavelength of $568 \mathrm{~nm}$ and at $6 \mathrm{~mW}$ of emitted power, was used to irradiate the MCA. The laser beam was positioned with a mirror and focused with a convex lens (Newport; KPX082AR.14) onto the MCA just distal to the cerebral vein. During laser irradiation, intravenous administration of a photosensitizing rose Bengal dye solution $(20 \mathrm{mg} / \mathrm{kg})$ over $90 \mathrm{~s}$ was commenced. Four minutes after irradiation, a Q-switched, frequency-tripled yttrium aluminum garnet (YAG) laser operating at $355 \mathrm{~nm}(16 \mathrm{~mW} ; 15 \mathrm{~Hz}$; average power, $2.3 \mathrm{~W} / \mathrm{cm}^{2}$ ) was focused with a $30 \mathrm{~cm}$ focal length cylindrical lens (CKX 300; Newport Corporation) and positioned with a mirror enveloping the occluded distal MCA.

Eye enucleation. Mice were anesthetized by intraperitoneal injection of ketamine $(0.13 \mathrm{mg} / \mathrm{g})$ and xylazine $(0.01 \mathrm{mg} / \mathrm{g})$, and the connective tissue around the sclera was carefully removed to expose the optic nerve. The nerve was then cut, both eyes were extracted, and the orbits were packed with sterile gelfoam (Prusky et al., 2006).

Statistical analysis. Values are the mean \pm SEM of independent experiments. Statistical differences were examined using one-way factorial ANOVA combined with Scheffe's test for all paired comparisons. Differences with $p$ values $<0.05$ were considered significant.

\section{Results}

\section{Resting microglial processes make brief contacts with synapses}

The motility of resting microglial cells were imaged in the intact brain using an Iba1-EGFP transgenic mouse. Microglial cells in 
A
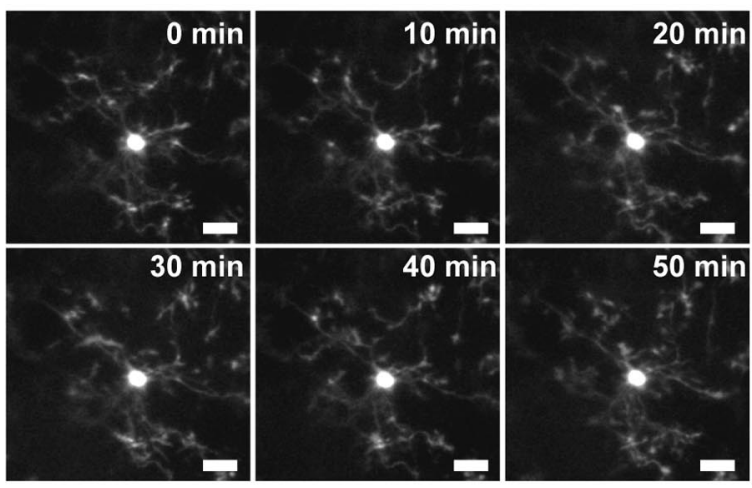

B

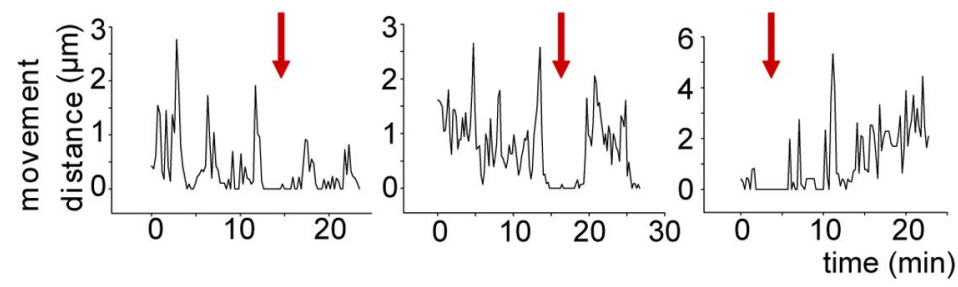

C
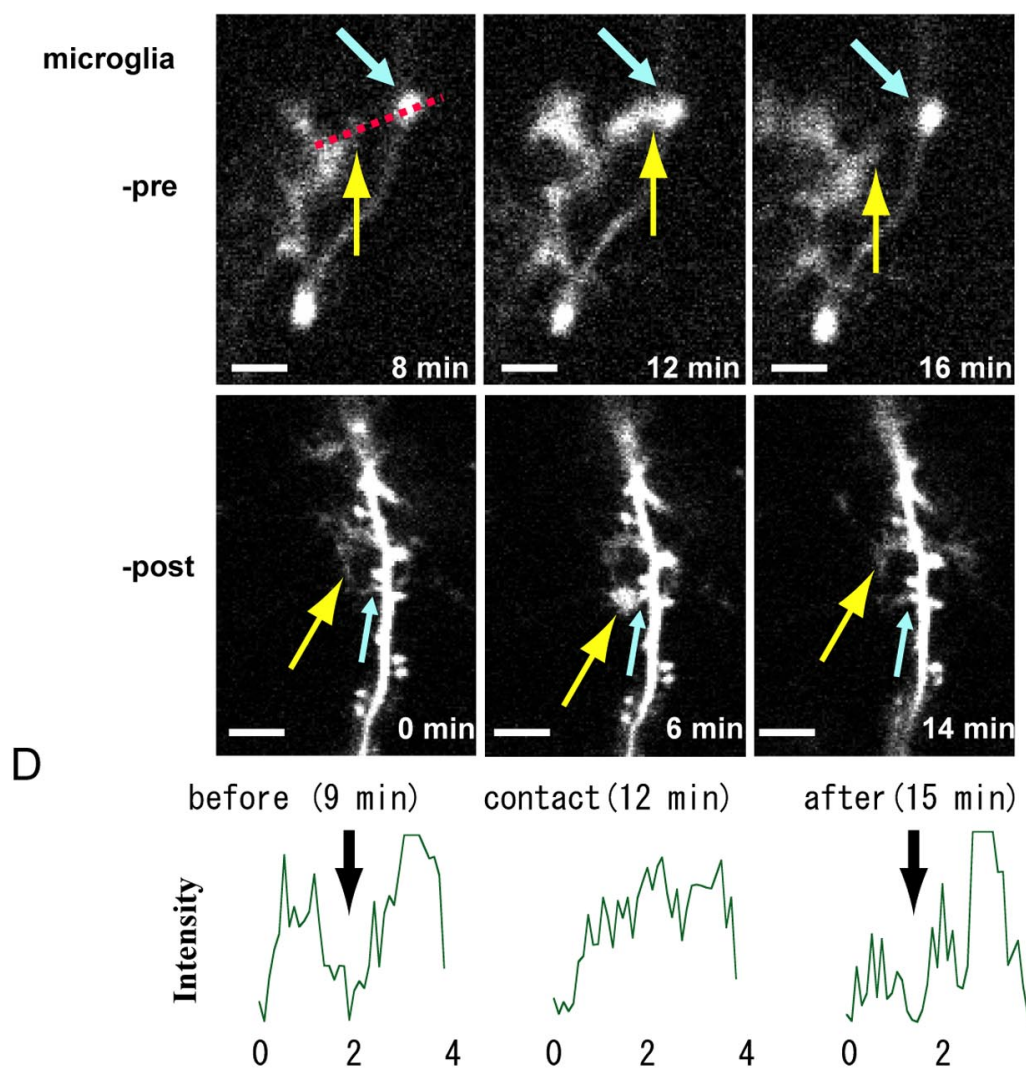

contact (12 $\mathrm{min})$
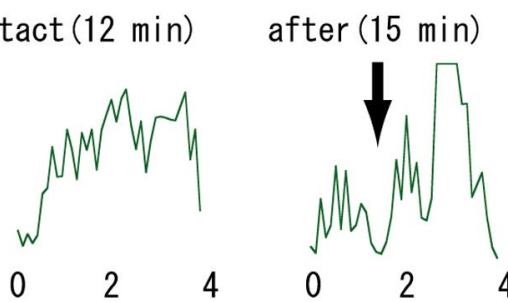

$4(\mu \mathrm{m})$

Figure 1. Dynamic motility of resting microglial processes and connection with synapses. $A$, Images of a single resting microglial cell in the intact cortex of an Iba1-EGFP mouse. Each is a stack image composed of $70-80$ focal planes, each advancing 0.5 $\mu \mathrm{m}$ along the $z$-axis. Scale bar, $20 \mu \mathrm{m}$. Resting microglia have extensive processes which extend in all directions. These processes are dynamic, moving back and forth. $\boldsymbol{B}$, Continuous monitoring of the motility of three representative microglial processes (from 3 different cells) using free-scanning imaging over $20 \mathrm{~min}$. The absolute position of each process was expressed relative to a fixed starting point and summed into $12 \mathrm{~s}$ bins. Note a brief $(\sim 4-5 \mathrm{~min})$ pause, indicated by the red arrows, in process motility. $C$ Contacts between microglial processes (yellow arrow in each image) and a presynaptic bouton (blue arrows in top row) and a postsynaptic spine (blue arrow in bottom row). Images in each row (-pre, -post) were obtained from single-plane free-scanning images at a higher resolution (scale bars: -pre, $2 \mu \mathrm{m}$; -post, $4 \mu \mathrm{m}$ ) (see supplemental Videos 1-Presynapse and 2-Postsynapse, available at www.jneurosci.org as supplemental material). Note that the tip of the microglial process is enlarged during the contact. Left, middle, and right panels in each row were obtained before, during, and after the contact of the microglial process vivo were imaged in layers II-III (from 150 to $250 \mu \mathrm{m}$ from the brain surface) of the somatosensory and visual cortex using two-photon microscopy (Denk and Svoboda, 1997). To avoid the potential mechanical tissue injury and activation of microglia (Xu et al., 2007), we used thin-skull transcranial imaging. As recently reported (Davalos et al., 2005; Nimmerjahn et al., 2005), we confirmed that the highly ramified processes of resting microglia are highly motile, without any accompanying movement of the cell soma (Fig. 1A). The speed and distance of microglial process retraction and extension was similar to each other, resulting in very little net displacement of resting microglial processes. To quantify more precisely the extent of this motility, we measured the movements of individual processes using continuous free scanning. There were brief static periods (typically $4-5 \mathrm{~min}$ ) before microglial processes resumed their extension-retraction motility (Fig. $1 B$ ).

It is possible that microglia make contacts with specific brain structures during static periods. To investigate possible contacts between microglial processes and neuronal elements of brain circuits, we crossbred the Iba1-EGFP mouse with a Thyl promoter GFP mouse (M-line) (Feng et al., 2000) and used line scanning to examine possible contacts between microglia and neuronal structures, and we focused specifically on synapses, the structures mediating neuronal communication (Fig. 1C). Presynaptic boutons and terminals are evident as bright spots along an axon resulting from the increased amount of GFP in presynaptic structures per unit length of axon as reported previously (De Paola et al., 2006). Contact between a microglial process and a presynaptic bouton was defined using the fluorescence intensity profile along a straight line between an identified bouton and process (Fig. 1C, broken red line, top left). When there was a gap between the bouton and process, there was a trough in the fluorescence in-

with the synaptic structure, respectively. $\boldsymbol{D}$, Plots of fluorescence intensity as a function of distance along a straight line between a bouton and adjacent microglial process, indicated by the red broken line in $\boldsymbol{C}$,-pre, left. The fluorescent intensity plot before contact (left) has two peaks, the left peak associated with the microglial process and the right with the presynaptic bouton. A region of minimum intensity, an intensity trough, is indicated by the black arrow and signifies a clear separation between the two structures. As the microglial process and the bouton came into contact, there was a loss of this fluorescence intensity trough (center). After the 5 min contact, the fluorescence intensity trough reappeared (right). 
A
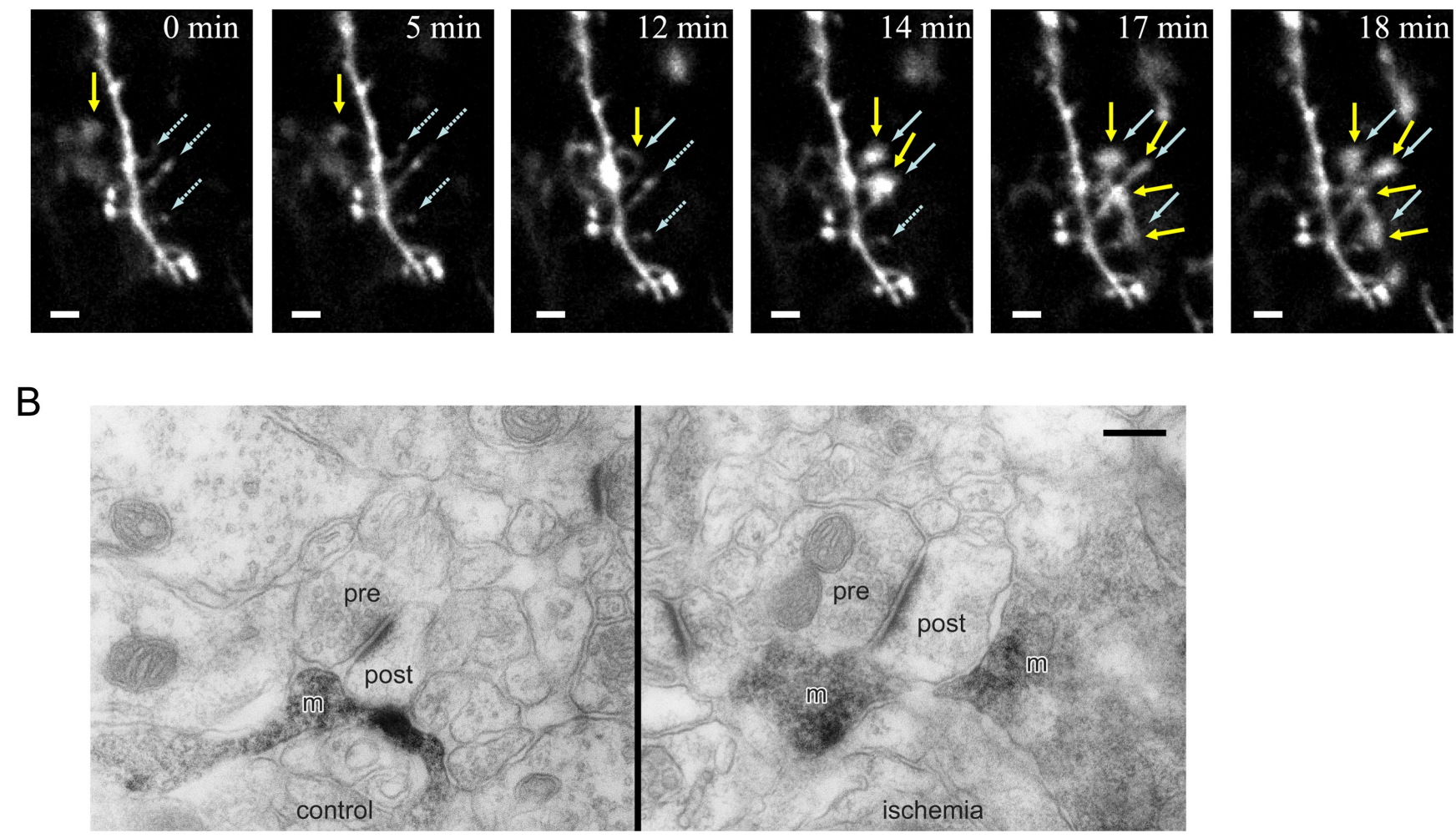

Figure 2. Direct and specific connection between microglia and synapses. $A$, Specific contacts between microglial processes (yellow arrow in each image), with postsynaptic dendritic spines (blue arrows in each image) along a single dendrite. The blue arrows indicating the spines are shown as solid arrows when contacted by the microglial process and as broken arrows when not in contact. Images were obtained from five planes of the $z$-axis of free-scanning images (scale bar, $2 \mu \mathrm{m}$ ). $\boldsymbol{B}$, Electron microscopic pictures of cortical slices obtained from control brains (left) and ischemic brains (right), illustrating the direct contact between presynaptic (pre)/postsynaptic (post) elements and adjacent microglial processes (m). After ischemia, the contacts appear more extensive. Scale bar, $200 \mathrm{~nm}$.

tensity profile, which disappeared during the contact (Fig. $1 D$, black arrows). As shown in Figures $1 C$ (microglia-pre) and supplemental Video 1 (Presynapse-microglia), available at www. jneurosci.org as supplemental material, the microglial processes briefly contacted presynaptic boutons, momentarily paused while in close apposition with the presynaptic structure, and then retracted. We quantified the contact duration using freescanning images (Fig. 1C; supplemental Video 1, Presynapsemicroglia, available at www.jneurosci.org as supplemental material) with contacts appearing as a fusion of the fluorescent signals from the reporter proteins in microglia and presynaptic terminals. The averaged duration of these contacts was $4.60 \pm 0.08 \mathrm{~min}$ (mean \pm SEM; 13 contacts, six mice). Time-lapse observations of individual presynaptic boutons revealed that the frequency of microglial contacts was $0.94 \pm 0.40$ contacts per hour (27 connections, six mice) (Fig. $3 B$ ) (body temperature, $37^{\circ} \mathrm{C}$ ). The microglial processes appeared to enlarge when in contact with the presynaptic terminals (supplemental Video 1, available at www. jneurosci.org as supplemental material).

\section{Microglia make specific and direct contact to synapses}

Bulbous contact between microglial process and dendritic spines were also observed, as shown in Figure $1 C$ (microglia-post) and supplemental Video 2 (Postsynapse-post synaptic spine), available at www.jneurosci.org as supplemental material. To investigate whether microglial processes randomly sampled neuronal domains, a serial image of a dendrite containing three closely located and sequential spines was obtained as shown in Figure $2 \mathrm{~A}$. A microglial cell extended its processes and made bulbous contacts with all three spines/synapses but did not make any contacts with the dendritic shaft. Thus, microglial processes preferentially and specifically contact synaptic structures. To confirm direct contact between microglia processes and synaptic structures, we used immuno-electron microscopic examination of serial cortical sections (see Materials and Methods). As shown in Figure $2 B$ (left panel; control), microglial processes made direct contacts with both presynaptic and postsynaptic structures.

\section{Activity-dependent modification of microglia-synapse contacts}

To investigate whether synaptic activity affects the microglial contact of synaptic structures, we used a number of simple approaches to regulate the basal neuronal activity, without direct accessing the cortex which could result in possible microglia activation. We imaged microglia in the visual cortex and deprived mice of any visual input by binocular eye enucleation. In separate experiments measuring intracellular $\mathrm{Ca}^{2+}$ transients, we confirmed that there was a reduction of basal neuronal activity in layer II/III of the visual cortex $2 \mathrm{~h}$ after binocular eye enucleation (supplemental Fig. 2, available at www.jneurosci.org as supplemental material). This extreme sensory deprivation resulted in a retraction of microglial processes over the subsequent $4-6 \mathrm{~h}$ (supplemental Video 3, available at www.jneurosci.org as supplemental material). We additionally used two other noninvasive approaches to modulate the spontaneous neuronal activity of visual cortex neurons: injection of tetrodotoxin (TTX) into both retina and a reduction of body temperature. In the separate experiments, we also confirmed, by measuring spontaneous $\mathrm{Ca}^{2+}$ transients, that both these procedures significantly reduced the basal activity of visual cortex neurons in vivo (Fig. $3 B, C$ ) (six 
animals each with 200 cells imaged) (Fig. $3 A)$. Under these conditions of reduced basal neuronal activity, the frequency of contacts between microglial processes and synapses was significantly reduced (Fig. $3 D$ ), being $0.48 \pm 0.10$ contacts per hour ( 12 connections, four mice) at $32^{\circ} \mathrm{C}$, and $0.37 \pm 0.12$ contacts per hour (seven connections, four mice) at $37^{\circ} \mathrm{C}$ in mice that had received TTX injections, compared with the control value $0.94 \pm$ $0.40 / \mathrm{h}$ at $37^{\circ} \mathrm{C}$. The reduced connection frequency at $32^{\circ} \mathrm{C}$ was not caused by a decrease in the speed of microglial process movements $(0.36 \pm 0.04 \mu \mathrm{m} / \mathrm{min}$ at $37^{\circ} \mathrm{C}$, four processes from four microglia and $0.32 \pm 0.04 \mu \mathrm{m} / \mathrm{min}$ at $32^{\circ} \mathrm{C}$, four processes from four microglia). Thus, reduced neuronal activity was associated with reduced contact frequency between synapses and microglia.

\section{Microglia-synapse connections are prolonged in response to ischemia} Microglial cells play neuroprotective and neuroregenerative roles in response to various pathological insults, involving both functional and morphological changes, e.g., cell and process migration and the release various neuroactive compounds (Vilhardt, 2005; Biber et al., 2007). To investigate how microglial-synapse communication may respond to pathological insults, we induced transient cerebral ischemia, using transcranial laser-induced photochemical occlusion of the left distal MCA (i.e., again without opening the cranial bone) (Yao et al., 2002). Microglia and neurons were visualized in the somatosensory cortex within the ischemic penumbra $(<0.5 \mathrm{~mm}$ lateral to the ischemic core). The contacts between microglial processes and presynaptic boutons were markedly prolonged by ischemia (Fig. $4 \mathrm{~A}$ ), from a very constant duration of $4.60 \pm 0.08 \mathrm{~min}$ (mean \pm SEM; 13 contacts, six mice) in control conditions, to $76.7 \pm 38.8 \mathrm{~min}$ in ischemia (12 connections, four mice). In addition to this prolonged contact time in ischemic brain, the microglial processes appeared to wrap more extensively around the synaptic structures (our unpublished observation), in contrast to the characteristic bulbous contact of microglia process with the presynaptic and postsynaptic structures in the nonischemic brain (Fig. 1C).

What may be the consequence of these prolonged contacts? In the healthy brain, the presynaptic boutons are very stable structures (supplemental Fig. $1 A, C$, available at www.jneurosci.org as supplemental material), in contrast to the markedly increased turnover rates in the ischemic penumbra (Zhang et al., 2005) (see also supplemental Fig. $1 B, C$, available at www.jneurosci.org as supplemental material). We, therefore, attempted to observe if the disappearance of synapses in ischemic brain could follow the prolonged contact by a microglial process. After transient isch- emia, more than half presynaptic boutons $(n=9$ of 12$)$ remained after prolonged contact with microglial process, whereas several contacted boutons ( 3 of 12 synapses observed) subsequently disappeared during or soon after the microglial contact (Fig. 4B).

\section{Discussion}

Recent studies have shown that, in the intact brain, the processes of resting microglia are highly motile, consistent with their proposed role as vigilant surveyors of the integrity and function of the brain (Davalos et al., 2005; Nimmerjahn et al., 2005). Furthermore, resting microglia may respond to, and repair, subclinical abnormalities of the brain without a complete activation transformation (Hanisch and Kettenmann, 2007). In the present study, we show that microglial processes make intimate but transient connections with neuronal synapses. Hence, the resting mo- 
A
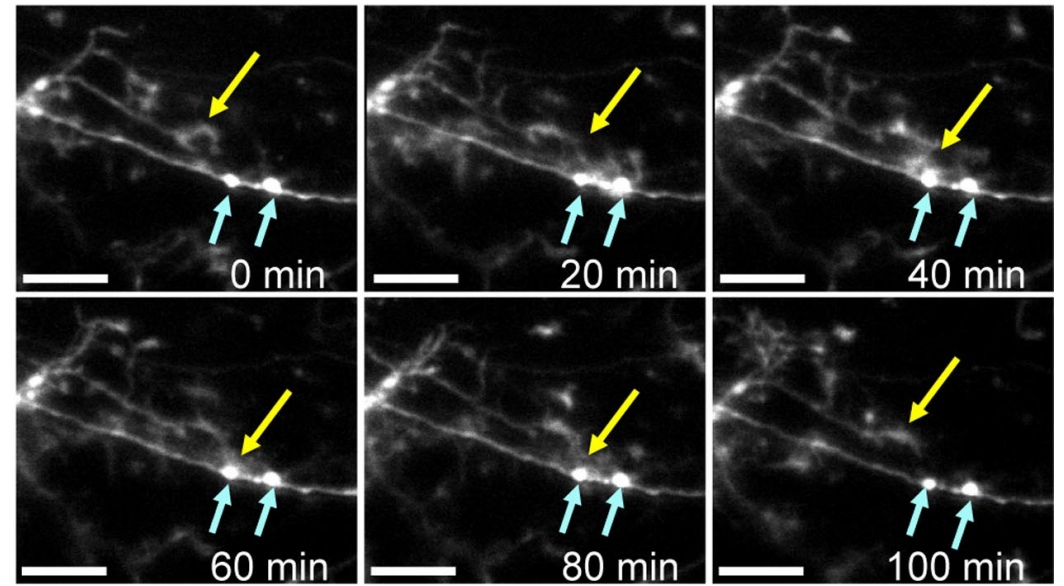

B
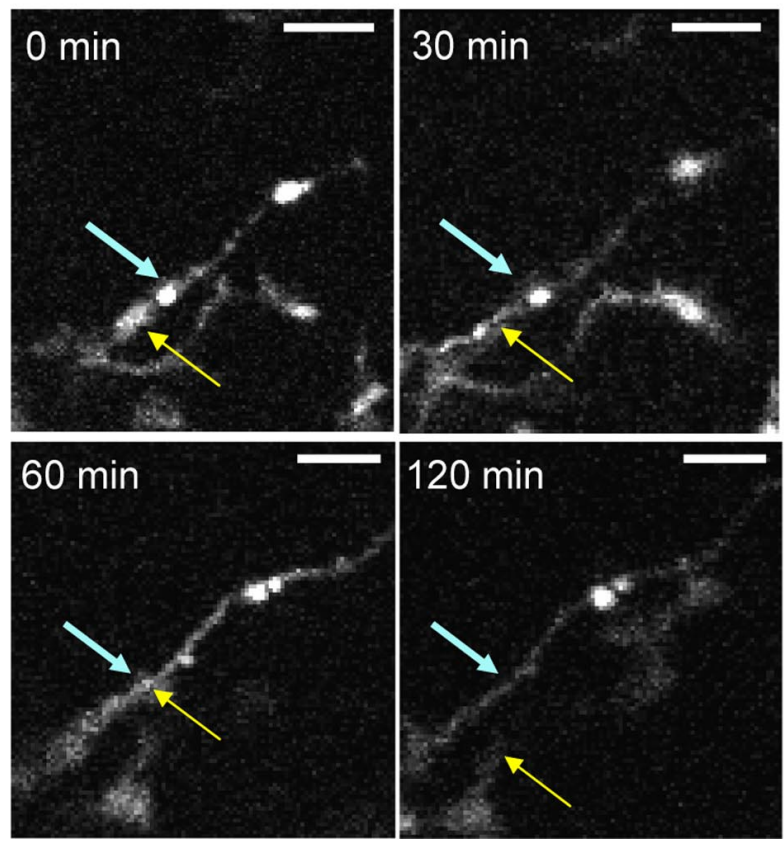

Figure 4. Prolongation of contacts between microglia processes and presynaptic boutons in response to cerebral ischemia and sequential loss of presynaptic bouton. $\boldsymbol{A}, \boldsymbol{z}$-stacked images of microglial processes (yellow arrow) and presynaptic boutons (blue arrows). Continuous images were acquired from the ischemic penumbra $\sim 500 \mu \mathrm{m}$ apart from the ischemic core and commenced $\sim 30$ min after MCA occlusion. Note that the contact between the microglial processes and the boutons lasted for $>60$ min, whereas it is very constant in duration about $5 \mathrm{~min}$ in the control brain (see text). In addition, the bulbous nature of the tip of the microglial processes once in contact with the pre(post)synaptic structures in the control (Fig. $1 B$ ) were not evident in the ischemic brains. Top left image shows a microglial process before contact, indicated as $0 \mathrm{~min}$, and bottom right shows the same process just after retraction. Scale bars, $5 \mu \mathrm{m}$. $\boldsymbol{B}$, The presynaptic bouton (indicated by the blue arrow) in the ischemic penumbra disappeared after the prolonged contact with a microglial process (yellow arrow). Note that the microglial process in this example crept forward and backward along the axon (top two panels) and gradually approached one of boutons (lower left bouton, blue arrow). Scale bars, $2 \mu \mathrm{m}$.

tility of microglial processes is not simply sampling freely diffusible signals in the extracellular space, but involves making direct connections with presynaptic and postsynaptic elements. The frequency of these connections was related to basal neuronal activity, with a connection time in the healthy brain that was strikingly constant, at $4-5 \mathrm{~min}$. This connection time corresponds well with the brief pauses in microglial process motility we observed (4-5 $\mathrm{min})$ and previously reported (3-4 $\mathrm{min})$ (Nimmerjahn et al., 2005). Contact with a synapse that is functioning normally may result in the induction of a repellant signal, or the reduced efficacy of an attractant signal, within this brief period and the microglia retract from the synapses. In contrast, the microglia-synapse connections persisted for an hour or more in the ischemic penumbra. We propose that microglia monitor and respond to the functional status of synapses, making brief contacts with healthy synapses and prolonged contacts with pathological or altered synapses.

The interactions between microglia and synapses may be mediated by a variety of chemo-attractant/repellant-signaling molecules and surface receptors. Possible candidate attractant signals which could be released from synapses include glutamate, ATP, brain-derived growth factor, and chemokines (Lessmann et al., 2003; Haynes et al., 2006), in addition to a role of any electrical signals. Microglia possess receptors for, and respond to, these substances (Nakajima et al., 1998; Noda et al., 2000; Pocock and Kettenmann, 2007). Indeed, the motility of resting and activated microglial processes is markedly affected by extracellular ATP (Davalos et al., 2005). Sustained contacts and functional communication between microglia and synapses may involve ligands and surface receptors. Microglia express their expression of both major histocompatibility complex (MHC) class I and class II antigens (Grenier et al., 1989) and increase its expression in response to various brain pathologies (Tooyama et al., 1990; Kreutzberg, 1996). More specifically, MHC I antigens have been proposed to play a role in the loss of synapses (Sabha et al., 2008). Our preliminary (unpublished) data indicate a reduction in MHC class I antigen expression in the ischemic brain, and mice with nonfunctional MHC class I antigens have a greater loss of synapses in response to axotomy (Cullheim and Thams, 2007). Secretion of cytokines as part of the immune response of activated microglia, such as TNF- $\alpha$ and IL-6, may also contribute to the destruction of synapses, as suggested for the loss of myelin in HIV patients (Tyor et al., 1993). On the contrary, an inhibition of TNF- $\alpha$ release by functional synapses, as observed in cocultures of neurons and microglia (Mott et al., 2004), may be one mechanism by which they are spared from elimination by microglia. Synaptic structures are very stable in the mature and intact control brain, but both dendritic spines (Zhang et al., 2005) and presynaptic terminals (current results) undergo an increased turnover rate for several weeks after ischemia, similar to the structural changes observed at synapses during development (Colman et al., 1997; Lendvai et al., 2000). In the present study, we have directly visualized presynaptic boutons disappearing after ischemia and after being in prolonged contact with microglia. We propose that prolonged microglial contact can initiate a cascade of events that results in synapse disappearance. Such a loss of 
synapses bears some resemblance to the loss of afferent synapses on to axotomized motoneurons ("synaptic stripping"), where proliferating microglia detach afferent terminals within a few days after nerve transaction (Blinzinger and Kreutzberg, 1968; Kreutzberg, 1996). Such synaptic stripping leads to a reduction of synaptic activity (Yamada et al., 2008) and a functional impairment of neuronal circuits (Graeber et al., 1993). Activated microglia have also been suggested to contribute to synaptic striping of connections to cortical neurons after an inflammatory lesion (Trapp et al., 2007). The precise sequence of events in microglia underlying this synaptic stripping is unclear, and loss of synapses in response to axotomy can still be observed when microglia proliferation and full activation is blocked (Kreutzberg, 1996; Kalla et al., 2001). The loss of synapses in the present study was coincident with contact with ramified-like microglia. Clearly, a variety of microglial morphological and functional phenotypes may be involved in the response to different neuronal injury.

In summary, we report direct, activity-dependent connections between microglia and synapses and have quantified the kinetics of this interaction. These interactions are very consistent in the control brain but markedly prolonged in the ischemic brain. We propose that microglia detect the functional state of the synapses, responding with prolonged contact under pathological conditions. Such prolonged contact is followed on some occasions by synapse elimination, suggesting that microglia are perhaps either attempting to restore synapse function or initiating their subsequent removal (as illustrated schematically in supplemental Fig. 3, available at www.jneurosci.org as supplemental material). Such microglial diagnosis of synapse function is likely to be important in any subsequent remodeling of neuronal circuits after brain injury and provides a novel target for the development of new therapies to improve the recovery of neuronal function after brain damage.

\section{References}

Bessis A, Béchade C, Bernard D, Roumier A (2007) Microglial control of neuronal death and synaptic properties. Glia 55:233-238.

Biber K, Neumann H, Inoue K, Boddeke HW (2007) Neuronal 'On' and 'Off' signals control microglia. Trends Neurosci 30:596-602.

Blinzinger K, Kreutzberg G (1968) Displacement of synaptic terminals from regenerating motoneurons by microglial cells. Z Zellforsch Mikrosk Anat 85:145-157.

Bruce-Keller AJ (1999) Microglial-neuronal interactions in synaptic damage and recovery. J Neurosci Res 58:191-201.

Colman H, Nabekura J, Lichtman JW (1997) Alterations in synaptic strength preceding axon withdrawal. Science 275:356-361.

Cullheim S, Thams S (2007) The microglial networks of the brain and their role in neuronal network plasticity after lesion. Brain Res Rev 55:89-96.

Davalos D, Grutzendler J, Yang G, Kim JV, Zuo Y, Jung S, Littman DR, Dustin ML, Gan WB (2005) ATP mediates rapid microglial response to local brain injury in vivo. Nat Neurosci pp 752-758.

Denk W, Svoboda K (1997) Photon upmanship: why multiphoton imaging is more than a gimmick. Neuron 18:351-357.

De Paola V, Holtmaat A, Knott G, Song S, Wilbrecht L, Caroni P, Svoboda K (2006) Cell type-specific structural plasticity of axonal branches and boutons in the adult neocortex. Neuron 49:861-875.

Feng G, Mellor RH, Bernstein M, Keller-Peck C, Nguyen QT, Wallace M, Nerbonne JM, Lichtman JW, Sanes JR (2000) Imaging neuronal subsets in transgenic mice expressing multiple spectral variants of GFP. Neuron 28:41-51.

Graeber MB, Bise K, Mehraein P (1993) Synaptic stripping in the human facial nucleus. Acta Neuropathol 86:179-181.

Grenier Y, Ruijs TC, Robitaille Y, Olivier A, Antel JP (1989) Immunohistochmical studies of adult human glial cell. J Neuroimmunol 21:103-115.

Hanisch UK, Kettenmann H (2007) Microglia: active sensor and versatile effector cells in the normal and pathologic brain. Nat Neurosci 10:1387-1394.

Haynes SE, Hollopeter G, Yang G, Kurpius D, Dailey ME, Gan WB, Julius D (2006) The P2Y12 receptor regulates microglial activation by extracellular nucleotides. Nat Neurosci 9:1512-1519.

Hirasawa T, Ohsawa K, Imai Y, Ondo Y, Akazawa C, Uchino S, Kohsaka S (2005) Visualization of microglia in living tissues using Iba1-EGFP transgenic mice. J Neurosci Res 81:357-362.

Hsu SM, Raine L, Fanger H (1981) Use of avidin-biotin-peroxidase complex $(\mathrm{ABC})$ in immunoperoxidase techniques: a comparison between $\mathrm{ABC}$ and unlabeled antibody (PAP) procedures. J Histochem Cytochem 29:577-580.

Kalla R, Liu Z, Xu S, Koppius A, Imai Y, Kloss CU, Kohsaka S, Gschwendtner A, Möller JC, Werner A, Raivich G (2001) Microglia and the early phase of immune surveillance in the axotomized facial motor nucleus: impaired microglial activation and lymphocyte recruitment but no effect on neuronal survival or axonal regeneration in macrophage-colony stimulating factor-deficient mice. J Comp Neurol 436:182-201.

Kreutzberg GW (1996) Microglia: a sensor for pathological events in the CNS. Trends Neurosci 19:312-318.

Lendvai B, Stern EA, Chen B, Svoboda K (2000) Experience-dependent plasticity of dendritic spines in the developing rat barrel cortex in vivo. Nature 404:876-881.

Lessmann V, Gottmann K, Malcangio M (2003) Neurotrophin secretion: current facts and future prospects. Prog Neurobiol 69:341-374.

Mott RT, Ait-Ghezala G, Town T, Mori T, Vendrame M, Zeng J, Ehrhart J, Mullan M, Tan J (2004) Neuronal expression of CD22: novel mechanism for inhibiting microglial proinflammatory cytokine production. Glia 46:369-379.

Nakajima K, Kikuchi Y, Ikoma E, Honda S, Ishikawa M, Liu Y, Kohsaka S (1998) Neurotrophins regulate the function of cultured microglia. Glia 24:272-289.

Nimmerjahn A, Kirchhoff F, Helmchen F (2005) Resting microglial cells are highly dynamic surveillants of brain parenchyma in vivo. Science 308:1314-1318.

Noda M, Nakanishi H, Nabekura J, Akaike N (2000) AMPA-kainate subtypes of glutamate receptor in rat cerebral microglia. J Neurosci 20:251-258.

Ohki K, Chung S, Ch'ng YH, Kara P, Reid RC (2005) Functional imaging with cellular resolution reveals precise micro-architecture in visual cortex. Nature 433:597-603.

Pocock JM, Kettenmann H (2007) Neurotransmitter receptors on microglia. Trends Neurosci 30:527-535.

Prusky GT, Alam NM, Douglas RM (2006) Enhancement of vision by monocular deprivation in adult mice. J Neurosci 26:11554-11561.

Sabha M Jr, Emirandetti A, Cullheim S, De Oliveira AL (2008) MHC I expression and synaptic plasticity in different mice strains after axotomy. Synapse 62:137-148.

Tooyama I, Kimura H, Akiyama H, McGeer PL (1990) Reactive microglia express class I and class II major histocompatibility complex antigens in Alzheimer's disease. Brain Res 523:273-280.

Trapp BD, Wujek JR, Criste GA, Jalabi W, Yin X, Kidd GJ, Stohlman S, Ransohoff R (2007) Evidence for synaptic stripping by cortical microglia. Glia 55:360-368.

Tyor WR, Glass JD, Baumrind N, McArthur JC, Griffin JW, Becker PS, Griffin DE (1993) Cytokine expression of macrophages in HIV-1-associated vacuolar myelopathy. Neurology 43:1002-1009.

Vilhardt F (2005) Microglia: phagocyte and glia cell. Int J Biochem Cell Biol 37:17-21.

Xu HT, Pan F, Yang G, Gan WB (2007) Choice of cranial window type for in vivo imaging affects dendritic spine turnover in the cortex. Nat Neurosci 10:549-551.

Yamada J, Hayashi Y, Jinno S, Wu Z, Inoue K, Kohsaka S, Nakanishi H (2008) Reduced synaptic activity precedes synaptic stripping in vagal motoneurons after axotomy. Glia 56:1448-1462.

Yao H, Okada Y, Ibayashi S (2002) Therapeutic time window for YAG laserinduced reperfusion of thrombotic stroke in hypertensive rats. Neuroreport 13:1005-1008.

Zhang S, Boyd J, Delaney K, Murphy TH (2005) Rapid reversible changes in dendritic spine structure in vivo gated by the degree of ischemia. J Neurosci 25:5333-5338. 